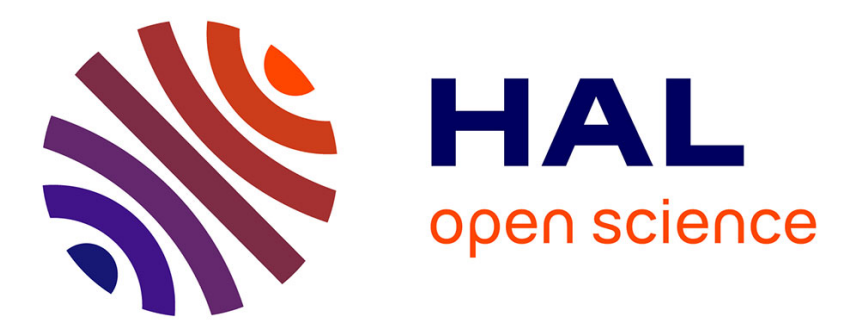

\title{
Les élections municipales en Italie: entre personnalisation, stabilisation de l'exécutif, coalitions et dé-nationalisation de la compétition
}

\author{
Camille Bedock
}

\section{- To cite this version:}

Camille Bedock. Les élections municipales en Italie: entre personnalisation, stabilisation de l'exécutif, coalitions et dé-nationalisation de la compétition. Groupement de recherches sur l'administration locale en Europe. Les élections locales françaises (2014-2015), 28, Le Moniteur, pp.247-253, 2016, Droit et Gestion des collectivités locales, 9782281132120. 10.3406/coloc.2016.2970 hal-02266796

\section{HAL Id: hal-02266796 https://hal.science/hal-02266796}

Submitted on 19 Aug 2019

HAL is a multi-disciplinary open access archive for the deposit and dissemination of scientific research documents, whether they are published or not. The documents may come from teaching and research institutions in France or abroad, or from public or private research centers.
L'archive ouverte pluridisciplinaire HAL, est destinée au dépôt et à la diffusion de documents scientifiques de niveau recherche, publiés ou non, émanant des établissements d'enseignement et de recherche français ou étrangers, des laboratoires publics ou privés. 
Les élections municipales en Italie : entre personnalisation, stabilisation de l'exécutif, coalitions et dé-nationalisation de la compétition

\section{Camille Bedock}

Publié dans Gérard MARCOU, Helmut WOLLMANN et Bernard DOLEZ, Droit et Gestion des collectivités locales 2016 : Les élections locales françaises (2014-2015). Paris : Le Moniteur.

Les élections municipales en Italie ont dans une très large mesure ouvert la voie à beaucoup des développements les plus marquants des institutions italiennes depuis 1993, mais aussi aux bouleversements permanents qu'a connu le système partisan italien depuis la même époque. En effet, les élections municipales ont été les premières en Italie à organiser l'élection directe du chef de l'exécutif local, et donc à promouvoir des logiques de personnalisation et de " présidentialisation de la vie politique $»^{1}$ présentes dans l'ensemble des démocraties consolidées, mais particulièrement prégnantes dans le cas de l'Italie. Comme le note ainsi Annick Magnier, l'Italie s'est positionnée à l'avant-garde d'un mouvement plus général, celui de l'élection directe du maire ${ }^{2}$. Ces développements institutionnels ont profondément changé le rôle du maire en lui donnant une prééminence nouvelle, mais les dynamiques institutionnelles municipales ne peuvent pas être dissociées des dynamiques électorales qui ont bouleversé le système partisan depuis 1993 de façon générale.

Ce chapitre précisera d'abord brièvement les modalités du système électoral en vigueur pour les élections municipales en Italie dans les régions à statut ordinaire. II reviendra ensuite sur les trois grands phénomènes caractérisant désormais la compétition électorale municipale en Italie en revenant sur le phénomène des primaires et sa généralisation, sur la stabilisation des exécutifs municipaux, et sur les dynamiques de coalition et de dé-nationalisation qui ont caractérisé la compétition municipale depuis 1993.

\section{I- $\quad$ Le système électoral italien pour les élections municipales}

La loi régissant l'élection directe des maires, mais aussi des présidents de province, du conseil communal et des conseils provinciaux est la loi du 25 mars $1993 n^{\circ} 81^{3}$, dite loi « Ciaffi ». Jusqu'au

\footnotetext{
${ }^{1}$ PoguntKe T. et P. WeBB (dir.), The Presidentialization of Politics: A Comparative Study of Modern Democracies, Oxford, Oxford University Press, coll.« Comparative politics », 2005.

${ }^{2}$ MAGNIER A., "Between Institutional Learning and Re-legitimization: Italian Mayors in the Unending Reform », International Journal of Urban and Regional Research, 1 mars 2004, vol. 28, $n^{\circ} 1$, p. 166-182.

${ }^{3} \mathrm{http}: / /$ www.ancitel.it/amministrative/allegati/Legge25031993n81.pdf
} 
début des années 1990, les exécutifs locaux ont été caractérisés par une grande instabilité due, selon Gianfranco Baldini à une "dépendance au niveau local des dynamiques de coalition nationales ". Cette situation se traduisait par une multiplicité de conflits entre des maires cherchant à affirmer leur autonomie au niveau local et leurs partis nationaux. L'Italie compte environ 8.000 communes, un nombre de très loin inférieur aux quelques 36.000 communes françaises. Avant 1992, seules les communes comptant moins de 5.000 habitants utilisaient un système électoral requérant une majorité absolue des voix, tandis que toutes les autres communes avaient un système électoral entièrement proportionnel qui aboutissait non seulement à un pouvoir extrême des partis pour contrôler le niveau local, mais aussi à un très faible niveau d'autonomie du maire ${ }^{5}$. Le maire était ensuite choisi par les conseillers municipaux eux-mêmes, dont la désignation répondait à une balance complexe entre coalitions locales et coordination avec le niveau national. La logique partisane qui présidait donc au niveau national, consistant à répartir sièges et responsabilités strictement en fonction de critères d'appartenance partisane et de stratégies de coalition, était donc reproduite fidèlement au niveau municipal.

La réforme de 1993, qui affecte les quinze régions ordinaires italiennes ${ }^{6}$ a introduit une rupture profonde, que certains qualifient même de "révolution ${ }^{7}$ par rapport à l'équilibre précédemment décrit. Le système mis en place peut être qualifié de simul stabent simul cadent impliquant que la chute de l'exécutif municipal implique aussi la dissolution de l'assemblée municipale, et réglemente l'élection directe du maire. Le système adopté diffère pour les communes comptant moins de 15.000 habitants et les $10 \%$ de communes en comptant davantage. Dans les communes de moins de 15.000 habitants a été mis en place un système pour l'élection du maire qui s'accompagne d'un bonus majoritaire très substantiel accordant au parti ou à la coalition ayant obtenu le plus de voix deux tiers des sièges dans le conseil municipal. Les maires obtiennent aussi la possibilité de recruter les assesseurs hors du conseil municipal lui-même ${ }^{8}$. Il est par ailleurs possible d'être à la fois adjoint au maire (assessore) tout en gardant son mandat de conseiller municipal (consigliere). Aucun seuil n'est prévu pour pouvoir accéder à la répartition des sièges au conseil municipal, les sièges n'ayant pas été gagnés par la liste arrivée en tête étant distribués selon la méthode d’Hondt. Les électeurs ont la

\footnotetext{
${ }^{4}$ BALDINI G., "The direct election of mayors: an assessment of the institutional reform following the Italian municipal elections of 2001 ", Journal of Modern Italian Studies, janvier 2002, vol. 7, n 3, p. 364.

${ }^{5}$ Ibid., p. 365.

${ }^{6}$ Il est à noter que les régions italiennes à statut spécial, à savoir la Sicile, la Sardaigne, le Val d'Aoste, le Frioul Vénétie julienne et le Trentin-Haut Adige utilisent des systèmes électoraux qui leur sont propres, bien que présentant de fortes similarités avec le système décrit ci-dessus.

${ }^{7}$ TARLI BARBIERI G., «I sistemi elettorali comunali anche alla luce delle elezioni del maggio 2015: ripensare la «rivoluzione» del 1993? ", Le Regioni, 2015, 3/2015, p. 705.

${ }^{8}$ BALDINI G., "The direct election of mayors », op. cit., p. 366.
} 
possibilité d'exprimer une préférence sur les listes municipales et les candidats ayant obtenu le plus de préférences sont élus au conseil.

Le système électoral prévu pour les communes de plus de 15.000 habitants présente plusieurs différences importantes par rapport à celui décrit ci-dessus: la possibilité d'émettre un vote "disgiunto » (disjoint) (1), la possibilité d'un candidat-maire d'être relié à plusieurs listes (2), la possibilité d'organiser un deuxième tour (3) et les modalités d'attribution du bonus majoritaire (4). Le système électoral dans les communes de plus de 15.000 habitants permet ainsi aux électeurs de formuler deux votes différents, en optant par exemple pour un candidat au poste de maire et une liste associée à un candidat différent. Les électeurs ont aussi la possibilité de ne pas voter pour le conseil municipal mais d'utiliser seulement leur vote pour désigner un candidat au poste de maire. Contrairement aux villes de moins de 15.000 habitants, il faut la majorité absolue et non pas relative pour l'emporter: dans le cas où aucun candidat à la position de maire n'a obtenu la majorité absolue des suffrages, un deuxième tour est organisé entre les deux candidats-maires ayant obtenu le plus de voix. Possibilité est alors données aux deux candidats de chercher le soutien des listes qui ont été éliminées. Contrairement aux communes de plus petite taille, il n'est pas possible d'être à la fois adjoint et conseiller, et le candidat-maire a la possibilité d'être lié non pas à une seule, mais à plusieurs listes. La prime majoritaire attribuée diffère également de celle prévue dans les petites communes. Si un candidat-maire est élu dès le premier tour, le ou les listes liées à ce candidat obtiennent alors $60 \%$ des sièges à condition d'avoir obtenu au moins $40 \%$ des suffrages exprimés et moins de $50 \%$ des suffrages exprimés. Si un deuxième tour est organisé, le candidat-maire et les listes liées à ce candidat obtiennent $60 \%$ des sièges. Les listes ayant obtenu moins de $3 \%$ des suffrages au premier tour et qui ne sont liées à aucun coalition ayant dépassé ce seuil ne peuvent obtenir aucun siège.

Une série de modifications sont venues amender les modalités prévue par la loi de 1993. Il est ainsi à noter qu'un maire ne peut exercer plus de deux mandats consécutifs de quatre ans, puis de cinq ans depuis la loi $n^{\circ} 120$ de 1999 et le décret loi du 18 août $2000^{9}$. Par ailleurs, la loi n²15 du 23 novembre 2012 a introduit la "doppia preferenza di genere " (double préférence de genre) qui stipule que dans les communes de plus de 5.000 habitants, l'électeur a désormais la possibilité d'exprimer non pas une mais deux préférences à condition que ces préférences soient donnés à des candidats de chaque sexe, sous peine $d^{\prime}$ invalidation $d u$ vote ${ }^{10}$. La même loi prévoit également que

\footnotetext{
9 Decreto legislativo "Testo unico delle leggi sull'ordinamento degli enti locali", 18 août 2000, http://www.camera.it/parlam/leggi/deleghe/00267dl.htm

${ }^{10}$ Legge $n^{\circ} 215$ "Disposizione per promuovere il riequilibrio delle rappresentenze di genere nei consigli e negli giunte degli enti locali e nei consigli regionali. Disposizioniin materia di pari opportunità nella composizione delle commissioni di concorso nelle pubbliche amministrazioni", 23 novembre 2012
} 
dans les communes comptant plus de 5.000 habitants, aucun des deux sexes ne peut être représenté par plus de deux tiers des candidats.

Cet ensemble de lois électorales doit aussi être relié à des dispositifs législatifs importants concernant le rôle des exécutifs locaux, leurs compétences et leur autonomie, et en particulier la loi $\mathrm{n}^{\circ} 142$ de $1990^{11}$. Cette loi permet par exemple au maire de choisir une partie des adjoints en dehors du conseil municipal, concentre la responsabilité exécutive dans les mains du maire et donne aux communes une autonomie statutaire.

Giovanni Tarli Barbieri qualifie ces réformes de "modèle néo-parlementaire ${ }^{12}$, considérant par ailleurs que la loi Ciaffi est à la fois un modèle déviant et paradigmatique par rapport à l'ensemble es lois électorales adoptées successivement en Italie : déviant parce que cette loi introduit un modèle de gouvernement local en forte rupture avec le modèle précédent, paradigmatique parce que le modèle de la prime majoritaire est ensuite devenu une modalité présente dans la plupart des réformes électorales successives, qu'elles touchent aux élections locales ou nationales. La loi constitutionnelle $n^{\circ} 1$ de 1999 introduit ainsi l'élection « directe » du président du Conseil régional. De même, le nouveau système électoral adopté en $2015^{13}$ reprend de nombreuses modalités de la loi Ciaffi : bonus majoritaire, organisation d'un deuxième tour opposant les deux candidats arrivés en tête et les listes qui leur sont rattachées, etc. ${ }^{14}$.

\section{II - La compétition municipale depuis 1993 en Italie : diffusion des primaires, stabilisation des exécutifs municipaux et logiques mouvantes de coalition}

La compétition municipale en Italie a connu trois grands bouleversements depuis 1993, liés de manière plus ou moins directe aux nouvelles dispositions institutionnelles mais aussi à des dynamiques politiques nationales et locales. En premier lieu, l'élection directe des maires a contribué à diffuser avec le temps de manière exponentielle le modèle des primaires dans un contexte plus général de personnalisation exacerbée de la vie politique italienne ${ }^{15}$ et de maintien depuis les années

\footnotetext{
http://www.gazzettaufficiale.it/eli/id/2012/12/11/012G0237/sg

${ }^{11}$ Legge $n^{\circ} 142$ "Ordinamento delle autonomie locali", 8 juin 1990 http://www.comune.jesi.an.it/MV/leggi/l142-90.htm

${ }^{12}$ TARLI BARBIERI G., "I sistemi elettorali comunali anche alla luce delle elezioni del maggio 2015 », op. cit., p. 507.

${ }^{13}$ Legge $n^{\circ} 52$ "Disposizioni in materia di elezione della Cammera dei deputati", 6 mai 2005, http://www.gazzettaufficiale.it/eli/id/2015/05/08/15G00066/sg

${ }^{14}$ BALDINI G. et A. RENWICK, «Italy toward (Yet Another) Electoral Reform », Italian Politics, 1 juin 2015, vol. 30, $\mathrm{n}^{\circ} 1$, p. $160-178$

${ }^{15}$ VENTURINO F. (dir.), Elezioni e personalizzazione della politica, Roma, Aracne editrice, 2005.
} 
1990 dans l'opinion publique italienne d'un très fort sentiment anti-partis ${ }^{16}$. Deuxièmement, les exécutifs municipaux ont désormais acquis une stabilité incomparable par rapport à la situation qui prévalait avant 1993. Troisièmement et enfin, la compétition politique au niveau local est devenue une bataille entre coalitions, bipolaire, puis tripolaire depuis 2011, qui s'est aussi accompagné d'une progressive « dé-nationalisation » de la compétition électorale municipale.

Le premier phénomène résultant indirectement du nouveau mode d'élection des exécutifs municipaux est celui de l'extraordinaire développement de l'outil des primaires, encore totalement marginal dans les années 1990 et désormais devenu incontournable à tous les niveaux de compétition électorale en Italie. Comme le notent Antonella Seddone et Marco Valbruzzi, ce phénomène est indissociable en Italie d'un contexte dans lequel les partis traversent à la fois une crise de légitimité, ont une capacité d'attraction en berne et une vulnérabilité de plus en plus grande en tant qu'organisation ${ }^{17}$. Les deux auteurs montrent qu'entre 2004 et 2011, 392 primaires municipales ont été organisées (dont 372 à l'initiative du centre-gauche), un chiffre en nette augmentation depuis 2007 et la naissance du Parti démocrate qui a entraîné sa diffusion au-delà du centre-gauche $^{18}$. Cet effet de contagion géographique et partisane a aussi amené à leur institutionnalisation ${ }^{19}$. La recherche sur le sujet a par ailleurs montré que ces primaires étaient dans bien des cas non seulement réellement compétitives, mais ont à l'occasion favorisé des candidatures qui déplaisaient au parti central et a favorisé des candidatures en dehors des grands partis, comme par exemple à Milan avec Giuliano Pisapia ${ }^{20}$. Ce phénomène et sa diffusion au niveau municipal doit donc être compris comme la confrontation de deux logiques : ce que Gianfranco Pasquino appelle "l'américanisation de la vie politique italienne ${ }^{21}$ (personnalisation et affaiblissement des appareils partisans) et la volonté d'une sélection plus "démocratique " et inclusive des candidats à tous les niveaux de compétition électorale.

Le second phénomène frappant caractérisant la compétition municipale en Italie depuis 1993 est la stabilisation des exécutifs municipaux. D'après Carlo Baccetti, le nouveau système électoral a eu pour principal conséquence « de placer des politiciens agissant individuellement (...) dans la position centrale qui était auparavant occupée par des politiciens agissant collectivement (les partis

\footnotetext{
${ }^{16}$ BARDI L., « Anti-party sentiment and party system change in Italy », European Journal of Political Research, 1 avril 1996, vol. 29, n 3, p. 345-363.

${ }^{17}$ Seddone A. et M. VAlbruzZI (dir.), Primarie per il sindaco: Partiti, candidati, elettori, Milano, Egea, 2012.

${ }^{18}$ Ibid.

19 SAndri G. et F. Venturino, "Primaries at the municipal level: how, how many and why ", Contemporary Italian Politics, 2016, vol. 8, $\mathrm{n}^{\circ} 1$, p. 62-82.

${ }^{20}$ Seddone A. et M. Valbruzzi (dir.), Primarie per il sindaco, op. cit.

${ }^{21}$ PASQUINO G., "Primary elections in Italy. An episode in institutional imitation ", Journal of Modern Italian Studies, 2011, vol. 16, n 5 , p. 682.
} 
politiques) ${ }^{22}$. La logique ayant présidé à l'adoption des différentes lois portant sur les élections municipales introduisaient en effet une rupture profonde à la fois dans les relations entre partis et maire, mais aussi en termes des pouvoirs dont bénéficie le maire. En net contraste avec la situation qui prévalait avant 1993, la nouvelle loi donne ainsi aux maires une " position de prééminence ${ }^{23}$ que l'on peut expliquer notamment par la nouvelle stabilité des exécutifs municipaux acquise grâce à la prime majoritaire et grâce aux nouvelles normes sur le lien entre maire et conseil municipal. Le contraste est en effet frappant: alors que la durée moyenne d'un conseil municipal dans les 95 chefs-lieux de province entre 1972 et 1989 était largement inférieure à deux ans avant la réforme ${ }^{24}$, le nouveau modèle implique que la " chute » du maire implique également celle de tout le conseil. Par conséquent, le nombre de dissolution de conseils municipaux a drastiquement chuté : entre 1993 et 2001, pour les élections concernant des chefs-lieux de province, seuls 38 conseils municipaux sur 238 ne sont pas allés au bout de leur mandat ${ }^{25}$. Les nouvelles dispositions institutionnelles ont aussi contribué à donner aux maires une plus forte visibilité et davantage de responsabilités devant les électeurs, ainsi que plus de pouvoirs de contrôler le parti au niveau local ${ }^{26}$. D’après Silvia Bolgherini cependant, la période d'austérité budgétaire traversée par l'Italie depuis 2007 a contribué à un phénomène de "recentralisation" par le biais du renforcement de structures intercommunales élues indirectement et de coupes budgétaires. L'effet combiné de ces mesures a contribué à affaiblir le rôle prééminent acquis par le maire ${ }^{27}$.

Le troisième phénomène frappant ayant caractérisé la compétition municipale en Italie depuis 1993 est le rôle nouveau acquis par les coalitions électorales pour structurer la compétition mais aussi la dé-nationalisation progressive de la compétition. La loi électorale adoptée en 1993 a été qualifiée par Andrea Manzella de première pierre marquant le passage d'une "République des partis " à une "République des coalitions " ${ }^{28}$. Son adoption se situe en effet dans le contexte de l'effondrement du système partisan de ce qu'on a appelé la "Première République ", et est pratiquement concomitante à l'adoption de la loi dite Mattarellum qui marque au niveau national le

\footnotetext{
22 Baccetti, C. "Italy" dans Heinelt H. et X. Bertrana, The Second Tier of Local Government in Europe: Provinces, Counties, Départements and Landkreise in Comparison, Routledge, 2012, p.164-184.

${ }^{23}$ BALDINI, G., "La prima prova del modello neoparlamentare : il governo delle città italiane nel decennio 19932002" dans CECCANTI S. et S. VASSALLO (dir.), Come chiudere la transizione: cambiamento, apprendimento e adattamento nel sistema politico italiano, Bologna, II Mulino, 2004, p.151.

${ }^{24}$ BALDINI G., "The direct election of mayors ", op. cit., p. 368.

${ }^{25}$ Ibid.

${ }^{26}$ BolgherINI S., "Can Austerity Lead to Recentralisation? Italian Local Government during the Economic Crisis ", South European Society and Politics, 3 avril 2014, vol. 19, n² 2, p. 193-214.

27 Ibid.

${ }^{28}$ Manzella A., Il Parlamento, Bologna, II Mulino, 2003, p. 83.
} 
passage d'un système purement proportionnel à un système mixte majoritaire ${ }^{29}$. Le système partisan italien depuis 1993 a couramment été qualifié de "bipolarisme fragmenté ", se caractérisant au niveau local comme au niveau national par un regroupement des forces politiques autour de deux grandes coalitions composées d'un grand nombre de forces politiques ${ }^{30}$. Si certains auteurs parlent désormais de crise de ce "bipolarisme imparfait " ${ }^{31}$, notamment en raison de l'émergence fulgurante du Mouvement Cinq Etoiles qui a profondément transformé le système partisan italien depuis les années $2010^{32}$, la compétition municipale est désormais structurée par une bataille entre coalitions plutôt qu'entre partis. Cependant, l'élection directe du maire et l'affaiblissement du rôle des partis nationaux au niveau local a aussi donné lieu au développement de listes personnelles et de " listes civiques » parfois non-partisanes. Ce phénomène est resté marginal jusqu'à la fin des années 1990, mais la situation a rapidement et radicalement changé dans la dernière décennie : alors que les listes locales dans les villes de plus de 15.000 habitants représentaient $13 \%$ des élus municipaux entre 1995 et 1999, celles-ci représentent entre 2010 et 2014 près de 34,1\% des élus municipaux (respectivement $2,1 \%$ et $9,9 \%$ si l'on ne compte que les listes totalement indépendantes des partis politiques nationaux $)^{33}$. Ce phénomène est encore plus marqué en 2015 , particulièrement dans les municipalités de taille moyenne, dans les régions où les listes régionalistes sont absentes et dans celles précédemment dominées par une forte sous-culture politique catholique ou communiste ${ }^{34}$.

Pour conclure, le système électoral municipal en Italie doit être compris dans une grande mesure comme un exemple archétypique des grands bouleversements institutionnels et électoraux qu'a connus I'Italie depuis 1993, et de la relation complexe entre leaders locaux et partis nationaux, dans un contexte d'affaiblissement des partis politiques.

\footnotetext{
${ }^{29}$ BEDOCK C., « Du mattarellum au porcellum : une comparaison des réformes électorales de 1993 et 2005 en Italie ", Pôle Sud, 2011, $n^{\circ} 34, n^{\circ} 1$, p. 27-44.

${ }^{30}$ ChiaRAmonte A., « Dal bipolarismo frammentato al bipolarismo limitato? evoluzione del sistema partitico italiano. », R. D'Alimonte et A. Chiaramonte (dir.), Proporzionale se vi pare. Le elezioni politiche del 2008, Bologna, II Mulino, 2010, p. 203-228.

${ }^{31}$ MINALDI G. et C. RIolo, "La crisi del "bipolarismo imperfetto" », Rivista di storia delle idee, 2013, vol. 2, n ${ }^{\circ} 1$, p. 8-22.

${ }^{32}$ CONTI N. et V. MEMOLI, "The Emergence of a New Party in the Italian Party System: Rise and Fortunes of the Five Star Movement ", West European Politics, 4 mai 2015, vol. 38, n 3, p. 516-534.

${ }^{33}$ VAMPA D., "The 2015 Regional Election in Italy: Fragmentation and Crisis of Subnational Representative Democracy ", Regional \& Federal Studies, 8 août 2015, vol. 25, n 4, p. 377.

${ }^{34}$ VAMPA D., "The 2015 Regional Election in Italy », op. cit.
} 
